\title{
Uso da escuma de esgoto como matéria prima para produção de biodiesel usando a técnica de microondas.
}

Francisco Gustavo Hayala Silveira Pinto* (Graduando em Química na Universidade do Estado do Rio Grande do Norte - UERN)

Anne Gabriella Dias Santos (Profa. Ad. do Dq e do PPGCN na Universidade do Estado do Rio Grande do Norte - UERN)

Luiz Di Souza (Prof. Ad. do Dq e do PPGCN na Universidade do Estado do Rio Grande do Norte UERN)

Hilquias Sabino Barros (Mestre em Ciências Naturais Ppela Universidade do Estado do rio Grande do

\author{
Norte - UERN) \\ *Email:Gustavo_sk13@hotmail.com
}

Resumo: A necessidade de se desenvolver novas fontes de energias menos poluentes e de menor custo, tem gerado um incentivo na produção do biodiesel por ser uma fonte de energia renovável. A escuma de esgoto se apresenta como uma boa opção para tal ação, pois pode ser encontrada nas estações de tratamentos de esgotos em grandes quantidades. Este trabalho tem como meta a obtenção do biodiesel através da escuma de esgoto utilizando o método de transesterificação in situ tradicional e com o auxílio do microondas. No primeiro momento parte da escuma foi submetida ao método Bligh\&Dyer para extração do material lipídico (ML). O processo de transesterificação convencional foi realizado durante 4 horas a uma temperatura de $70{ }^{\circ} \mathrm{C}$ usando 10 gramas da escuma seca $50 \mathrm{~mL}$ de hexano, $150 \mathrm{~mL}$ etanol e $5 \%$ de $\mathrm{H}_{2} \mathrm{SO}_{4}$, as mesmas quantidades de reagentes fora utilizadas para a reação no microondas, com apenas uma exceção, onde o tempo de total da reação foi reduzido de 4 horas para 30 minutos e agitação constante, em seguida o material foi levado a um funil de decantação e separado solvente de material extraído, por fim o biodiesel obtido foi levado a estufa para eventual separação de solvente presente no meio. Após a extração foram feitas as análises de caracterização, por análises térmicas TG e DTG. O modelo do equipamento utilizado é o analisador térmico marca STA 449F3-J úpiter. O método de transesterificação in situ com a catalise ácida foi realizada com sucesso, a mesma técnica de extração se mostra eficaz na conversão da escuma em biodiesel, assim como o reação realizada com o auxílio do microondas se mostrou bastante promissora.

Palavras-chave: Transesterificação in situ; Biodiesel; Escuma; Microondas; Caracterização.

Espaço reservado para organização do congresso. 


\section{I NTRODUÇÃO}

Que os combustíveis fósseis são os mais utilizados para produção de energia para automóveis, isso todos já sabem, porém, só que junto a essa energia produzida vem as consequências, que são as emissões de gases poluentes que acabam por degradar o ambiente em que vivemos (BARROS; MAIA; SOUZA, 2013). Outra restrição para o uso dessa fonte de energia é o fato dela ser limitada, pois a mesma não é renovável, daí a preocupação de se achar meios viáveis de se produzir energia sem que aja uma degradação do ambiente em que vivemos e que essa mesma fonte de energia seja renovável.

A produção de biodiesel no Brasil é derivada majoritariamente da soja, sendo que, o emprego desta matéria prima corresponde a $70-85$ \% do custo total da produção de biodiesel. Esse custo tem influenciado diretamente no valor comercial do biodiesel que no Brasil, seu crescimento tem ocorrido de forma lenta. No ano de 2005, foi promulgada a obrigatoriedade da adição de $2 \%$ e permitiu a adição de 5 \% deste biocombustível ao diesel de petróleo. Em 2010, foi que se obrigou a adição dos 5 \% e somente em 2014 esta adição passou para os 7 \%. Em 9 anos, houve o incentivo de implementar apenas $6 \%$ de biodiesel, dificuldade está impulsionada por vários motivos, dentre eles o alto custo na sua produção (BARROS, 2015).

Visando encontrar uma forma de se produzir biocombustíveis que sejam economicamente mais viáveis as pesquisas partiram em busca de novas matérias primas para produção de biodiesel. Uma nova matéria prima que está sendo estudada é a escuma de esgoto presente nas estações de tratamento de esgoto. Durante as etapas de tratamento de esgotos se formam as escumas, as mesmas se formam devido a presença de gorduras provindas dos descartes de óleos vegetais e outros alimentos gordurosos (BARROS; MAIA; SOUZA, 2013; OLIVEIRA; BIESEK; PERGHER, 2014). Barros. Maia e Souza (2013) ainda diz que a produção de esgoto é em média 200L por pessoa e que desse, 160g é de escuma, no qual $10 \%$ seria gorduras. A produção de biodiesel a partir da escuma de esgotos é uma inovação, no que se desrespeita a produção de biodiesel (OLIVEIRA, 2004), assim pesquisas sobre a produção de biodiesel a partir da escuma de esgotos são bastantes limitadas.

Um dos principais entraves enfrentados na expansão e produção de biodiesel no Brasil, está relacionado, diretamente, com o seu custo final, ocasionado pela matéria prima utilizada ou pelo processo empregado na sua produção. Como forma de reduzir seu custo, uma série de esforços tem sido realizada, sendo que a transesterificação in situ aparece como um modelo de simplificação do processo de síntese do biodiesel pelo fato de descartar uma das etapas do processo (a extração do óleo), o que resulta em redução significativa no custo final do biodiesel (KARGBO, 2010; MONDALA et al., 2009; POKOO-AIKINS et al., 2010). A Figura 1 a seguir demonstra o mecanismo da reação. Outro grande interesse para redução dos custos e aumento de produção de biodiesel, são as reações realizadas com o auxílio do forno microondas,visando que as reações ocorrem em um menor intervalo de tempo, sendo que as reações realizadas nos mesmos tem uma maior eficiência (SHELTAWY, 2010, p. 310).

$\mathrm{O}$ uso do microondas para reações de transesterificação tem grandes vantagens quando comparadas com as reações convencionais visto que o tempo total da reação é consideravelmente inferior ao metodo convencional, (YUAN; YANG; ZHU, 2009), o uso de microondas ainda apresenta outras vantagens tais como, o método de aquecimento ser mais eficaz, economia da energia necessária e mais fácil de se ter o controle da reação (HUANG; WU; ZHU, 2013).

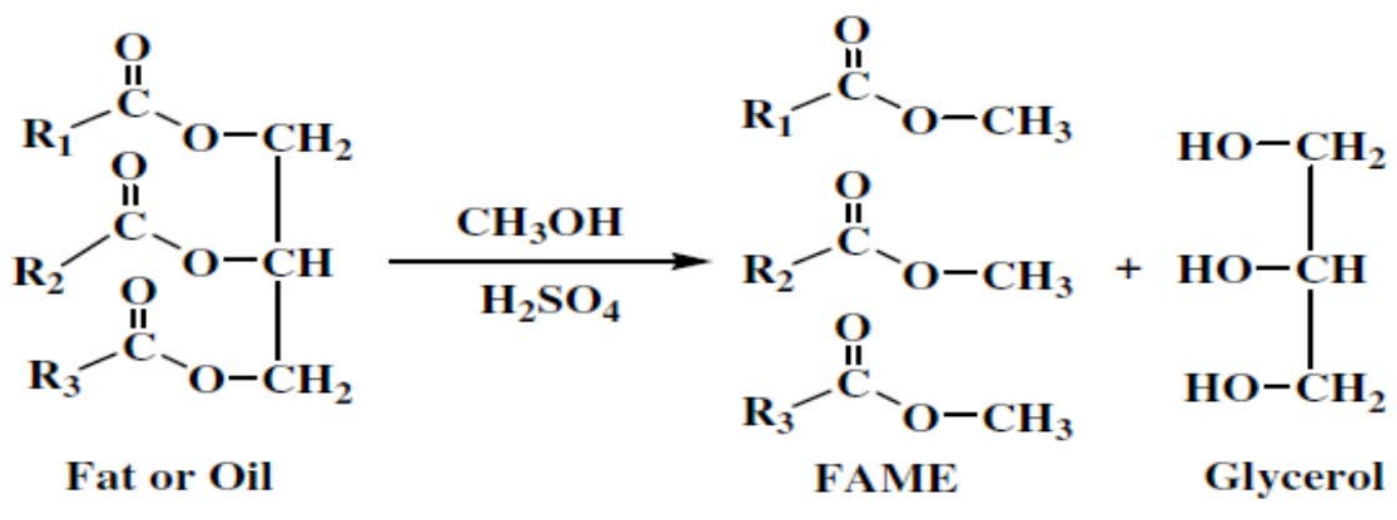


Figura 1. Mecanismo de reação de transesterificação de biodiesel.

Fonte: Mondala et al., 2009.

Desta forma, o presente trabalho tem como objetivo caracterizar a escuma de esgoto produzida na estação de tratamento de esgoto do Baldo em Natal/RN e analisar a viabilidade do seu uso tecnológico para produzir biodiesel via extração do material lipídico e sua transesterificação ou transesterificação in situ da escuma seca.

\section{METODOLOGIA}

\subsection{Extração do óleo}

A matéria prima foi coletada na estação de tratamento do baldo. Após isto houve a purificação dos resíduos, no qual a escuma foi desidratada em uma estufa a $110^{\circ} \mathrm{C}$ por 24 horas. Após a etapa de purificação a escuma passou a ter um aspecto pastoso. O método utilizado para extração do material lipídico foi o Bligh \& Dyer, no qual utilizou-se 50 gramas da amostra com as soluções dos solventes constituídas por $125 \mathrm{~mL}$ de clorofórmio, $250 \mathrm{~mL}$ de etanol e $50 \mathrm{~mL}$ de água agitadas por 30 minutos. Tal procedimento foi realizado para que se houvesse uma forma de comparar a TG/DTG do material lipídico puro com o material extraído pelo processo de transesterificação in situ convencional e com o auxílio do microondas.

\subsection{Transesterificação in situ}

Para a reação de transesterificação in situ pegou-se a escuma seca e pesou-se 10 gramas, junto a escuma foram adicionados $5 \%$ do $\mathrm{H}_{2} \mathrm{SO}_{4}, 150 \mathrm{~mL}$ de etanol e $50 \mathrm{~mL}$ de hexano. Em seguida a mistura foi mantida sob agitação e aquecimento $\left(70{ }^{\circ} \mathrm{C}\right)$ por 4 horas. Por fim a mistura foi filtrada e posta na estufa a $70{ }^{\circ} \mathrm{C}$ para remoção do solvente. Na transesterificação in situ utilizando o microondas foram repetidas as mesmas condições, com exceção ao uso do forno microondas e menor tempode reação que foi de 30 minutos para o método aqui mencionado. $\mathrm{O}$ forno microondas utilizado foi da Milestone, modelo Start Synth.

\subsection{Caracterização do material}

Após as reações foram feitas as análises de caracterização, por análises térmicas TG e DTG. Para isso, foi empregando um analisador térmico marca STA 449F3-Júpiter, com variação de temperatura de 30 a $600{ }^{\circ} \mathrm{C}$ e razão de aquecimento de $10{ }^{\circ} \mathrm{C} / \mathrm{min}$, sob atmosfera inerte de nitrogênio utilizando cadinho de alumina e aproximadamente $5 \mathrm{mg}$ da amostra. A técnica de TG/DTG foi realizada com a finalidade de se comparar a conversão do material lipídico em ésteres metílicos.

Para se determinar a acidez adicionou-se 0,2 g da amostra a um erlenmeyer de $125 \mathrm{~mL}, 25 \mathrm{~mL}$ de solução neutra de éter etílico e álcool etílicos na proporção de 2:1 respectivamente, a mistura foi mantida sob agitação para que ocorresse a homogeneização Adicionou-se 2 gotas de indicador verde de bromocresol e titulou-se com uma solução previamente padronizada de hidróxido de potássio a $0,01 \mathrm{~N}$ até que se obteve a coloração azul. Também foi realizado o teste com um branco repetindo o processo com todos os reagentes, exceto a amostra e obteve-se o índice de acidez por meio da equação 1.

$$
\begin{array}{ll}
\text { I.A. }=\frac{(V a-V b) \times N \times 5,61}{m} & \text { Equação } 1
\end{array}
$$

Onde: (I.A.) é o índice de acidez; (Va) é o volume em ML da solução de KOH a 0,01 gasto na titulação da amostra; (Vb) é o volume em $\mathrm{mL}$ da solução de $\mathrm{KOH}$ a $0,01 \mathrm{~N}$ gasto na titulação da solução (branco); N é a concentração da solução de KOH obtida na padronização da mesma; (m) é a 
massa em g da amostra e 5,61 é o fator de correção estequiométrica. O resultado final obtido foi expresso em $\mathrm{mg}$ de $\mathrm{KOH} / \mathrm{g}$ de amostra.

Para a determinação dos ácidos graxos livres foram adicionados a um erlenmeyer, 0,05g da amostra, $50 \mathrm{~mL}$ de álcool etílico a $95 \%$ previamente neutralizado com solução de $\mathrm{NaOH}$, aproximadamente 0,01 M. Em seguida, aqueceu-se a solução sobre uma placa térmica até iniciar a ebulição. Utilizaram-se duas gotas de indicador verde de bromocresol e titulou-se a quente com solução aquosa de $\mathrm{NaOH}$ aproximadamente $0,1 \mathrm{~N}$, até a coloração azul persistir por 15 segundos. O cálculo do teor de ácidos graxos livres baseou-se na equação 2 (SANTOS, 2010)

$$
\% \mathrm{AGl}=\underline{(\mathrm{Va}-\mathrm{Vb}) \times \mathrm{f} \times 28}
$$

Equação 2

m

Onde: (\% AGL.) é o índice de ácidos graxos livres; (Va) é o volume em $\mathrm{mL}$ da solução de $\mathrm{NaOH}$ a $0,01 \mathrm{~N}$ gasto na titulação da amostra; (Vb) é o volume em $\mathrm{mL}$ da solução de $\mathrm{NaOH}$ a $0,01 \mathrm{~N}$ gasto na titulação da solução (branco); f é o fator da solução de hidróxido de potássio; 28 é o equivalentegrama do $\mathrm{NaOH}$ e (m) é a massa em g da amostra.

\section{RESULTADOS E DISCUSSÃO}

\subsection{Extração do óleo}

O método Bligh \& Dyer apresentou boa quantidade de ML extraído (5,94 g), foram feitas apenas as principais caracterizações para o óleo obtido a partir da escuma devido à pouca quantidade que se tinha. A Tabela 1 nos apresenta os valores obtidos para o óleo obtido a partir da escuma

Tabela 1- Caracterização físico-química do óleo da escuma.

\begin{tabular}{cc}
\hline Características & Escuma \\
\hline Índice de acidez (mg KOH/g) & 60,03 \\
Ácidos graxos livres (\%) & 48,95 \\
\hline
\end{tabular}

O material obtido apresentou uma coloração escura e de cheiro forte, e sua viscosidade variou de um líquido viscoso a uma pasta. Essa aparência pode variar de acordo com a técnica de extração utilizada e a temperatura em que o ML é armazenado. Apesar da cor e a aparência física do óleo não ser considerada como parâmetro principal, propicia um indicativo da possibilidade de seu uso (CHRISTOFF, 2006), sendo a aparência viscosa um indicativo de maiores dificuldades no processo de síntese.

Os elevados valores registrados para as medidas de ácidos graxos livres e índice de acidez, aliada a elevada viscosidade do ML foram parâmetros que indicaram que a síntese para extração do com o ML não seria o mais adequado. O elevado valor de acidez pode ser devido ao fato de a matriz analisada ser constituída de óleos já utilizadas e descartados, tendo assim o mesmo já passado por uma degradação termoxidativa.

\subsection{Transesterificação tradicional}

É possível notar uma diferença nas quantidades de massas que se perde a medida que a temperatura vai variando, comparando a $\mathrm{TG}$ da matéria prima da escuma $\left(\mathrm{MP}_{\mathrm{E}}\right)$ com o material lipídico da escuma $\left(\mathrm{ML}_{\mathrm{E}}\right)$, conforme o Gráfico 1. 


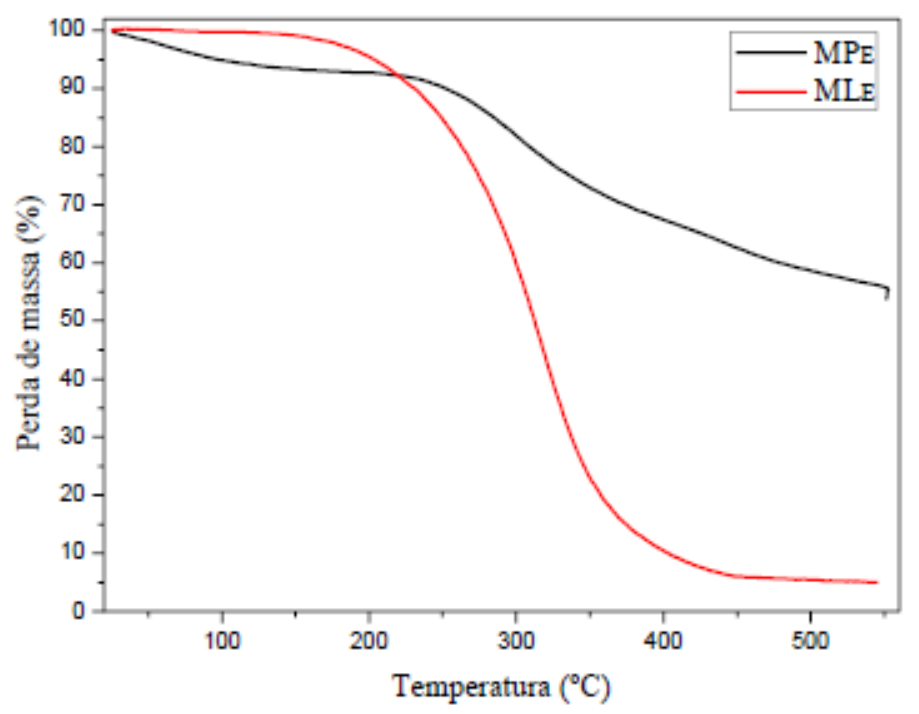

Gráfico 1. Analise físico-química da TG para a $\mathrm{MP}_{\mathrm{E}}$ e $\mathrm{ML}_{\mathrm{E}}$

É possível notar que para a MPe houve uma perda de massa de aproximadamente $41 \%$. Essa perda de massa é pequena se comparada com a do MLe que é de aproximadamente $94 \%$, vale lembrar a matéria prima se encontra em estado sólido e que o mesmo possui em sua composição materiais que não são orgânicos, esses materiais não orgânicos não sofrem degradação em temperaturas a baixo de $550^{\circ} \mathrm{C}$. Porém a MPe sofre duas perdas de massa uma de $30^{\circ} \mathrm{C}$ até $120^{\circ} \mathrm{C}$, no qual SILVA, $2010 \mathrm{diz}$ ser devido a presença de água residual e materiais de baixa massa molar e apresenta ainda uma outra perda de massa numa faixa de temperatura de $220^{\circ} \mathrm{C}$ a $450^{\circ} \mathrm{C}$ na qual se atribui a degradação dos materiais orgânicos (GARCIA et al., 2007, GOMES, 2013). Já o MLe apresenta apenas uma perda de massa, onde acredita-se que seja apenas devido a presença de compostos orgânicos já que a faixa onde essa perda ocorre é de $220^{\circ} \mathrm{C}$ a $450{ }^{\circ} \mathrm{C}$.

Para facilitar a visualização dos eventos ocorridos segue o gráfico da DTG tanto do MLe como do MPe, é clara a diferença nas intensidades dos picos sendo a do MLe bem mais fácil de visualizar. Ainda é possível enxergar a presença de um terceiro pico na amostra MPe, esse evento começa aproximadamente na temperatura de $394{ }^{\circ} \mathrm{C}$ e vai até $506^{\circ} \mathrm{C}$.

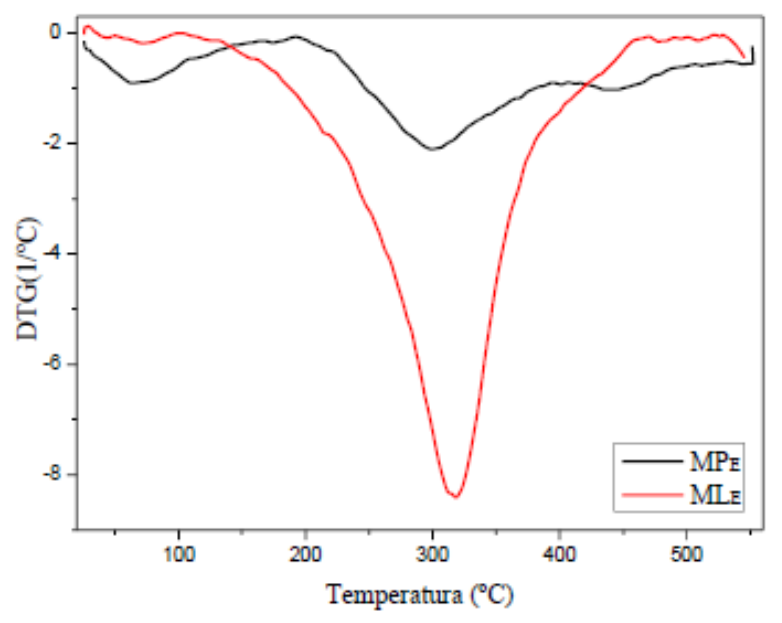

Gráfico 2. Análise de DTG para $\mathrm{MP}_{\mathrm{E}}$ e $\mathrm{ML}_{\mathrm{E}}$.

Como é possível ver ambos os materiais apresentam uma perda de massa em uma temperatura próximo a $100{ }^{\circ} \mathrm{C}$ o que poderia ser atribuído a perda de água presente nos materiais analisados ou então a volatilização de compostos de baixa massa molecular. A faixa na qual ocorre uma perda de massa mais acentuada é entre as temperaturas de $100{ }^{\circ} \mathrm{C}$ a $470{ }^{\circ} \mathrm{C}$, Dweck et al. (2004) e Gomes 
(2013) atribuem essas perdas de massa a decomposição ou volatilização de triacilglicerídios ou decomposição de compostos semelhantes.

Na Tabela 2 está descrito os dados termogravimétricos para o MPe e MLe, no caso do MLe pelo fato do segundo e terceiro ponto serem bem próximos um do outro ambos foram unificados a apenas uma faixa de temperatura.

Tabela 2- Faixa de temperatura onde se ocorre as principais perdas de massa para o $\mathrm{ML}_{\mathrm{E}}$ e $\mathrm{MP}_{\mathrm{E}}$.

\begin{tabular}{cccccc}
\hline AMOSTRAS & EVENTOS & $\mathbf{T}\left({ }^{\circ} \mathbf{C}\right)$ & Tmáx. $\left({ }^{\circ} \mathbf{C}\right)$ & $\begin{array}{c}\text { PERDA DE } \\
\text { MASSA (\%) }\end{array}$ & $\begin{array}{c}\text { MASSA } \\
\text { RESIDUAL }\end{array}$ \\
\hline \multirow{2}{*}{ MPe } & 1 & $26-192$ & 65 & 6,93 & \\
& 2 & $192-394$ & 299 & 24,88 & 58,53 \\
\multirow{2}{*}{ MLe } & 3 & $394-506$ & 439 & 9,62 & \\
& 1 & $25-101$ & 73 & 0,44 & 5,63 \\
\hline
\end{tabular}

No Gráfico 3 é possível notar a presença de dois ombros no pico principal. Considerando que houve três eventos principais para o biodiesel da escuma onde o primeiro se inicia $113{ }^{\circ} \mathrm{C}$ e vai até $255^{\circ} \mathrm{C}$, se compararmos com a DTG do MLE pode-se notar que o mesmo não se encontra podendo assim ser considerado como biodiesel produzido na reação. O segundo pico que ocorre na faixa de 255 ${ }^{\circ} \mathrm{C}$ a $376{ }^{\circ} \mathrm{C}$ é considerado como ML não reagido durante a reação e por fim o terceiro pico que vai de $376{ }^{\circ} \mathrm{C}$ a $490{ }^{\circ} \mathrm{C}$ é devido a presença de materiais de maior massa molar, podendo ser material não reagido, biodiesel de maior massa molar ou até mesmo biodiesel com estrutura diferente da que foi identificada no evento anterior.

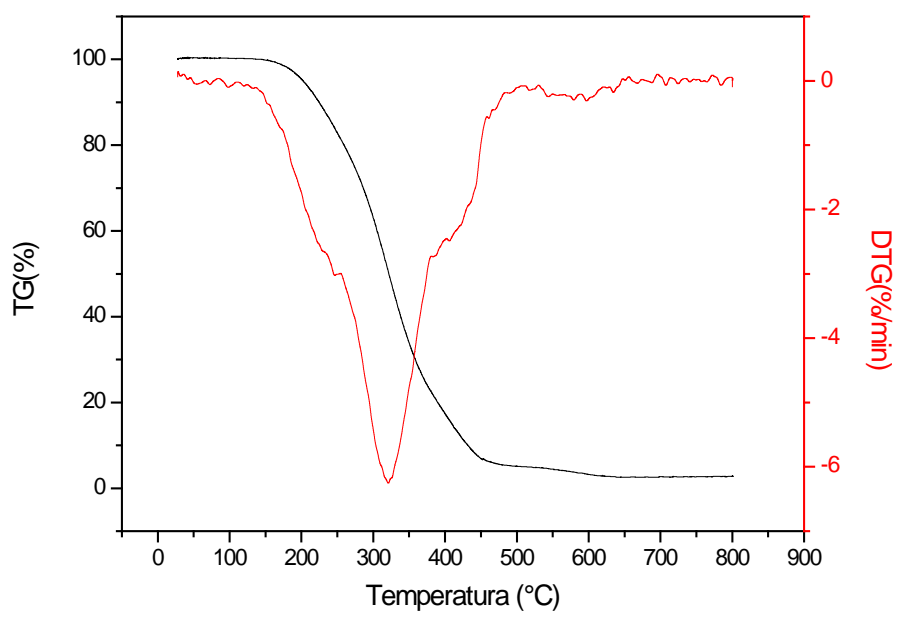

Gráfico 3. TG e DTG para a biodiesel produzido a partir da escuma de esgoto.

A Tabela 3 consta os intervalos das temperaturas de cada um destes eventos (Temperatura inicial e final), bem como temperatura máxima de cada evento.

Tabela 3- Faixa de temperatura onde se ocorre as principais perdas de massa para o biodiesel da escuma de

\begin{tabular}{ccccc}
\hline EVENTOS & T( $\left.{ }^{\circ} \mathbf{C}\right)$ & T Max $\left({ }^{\circ} \mathbf{C}\right)$ & $\begin{array}{l}\text { PERDA DE } \\
\text { MASSA (\%) }\end{array}$ & $\begin{array}{l}\text { MASSA } \\
\text { RESIDUAL } \\
\text { (\%) }\end{array}$ \\
\hline 1 & & & & 5,80 \\
2 & $113-255$ & 246 & 18,66 & \\
3 & $255-376$ & 321 & 57,70 & \\
4 & $376-490$ & 420 & 10,56 & \\
\hline
\end{tabular}




\subsection{Biodiesel produzido no Microondas}

No Gráfico 4 pode-se analisar a TG e DTG realizada para a transesterificação in situ realizado com a escuma no microondas.

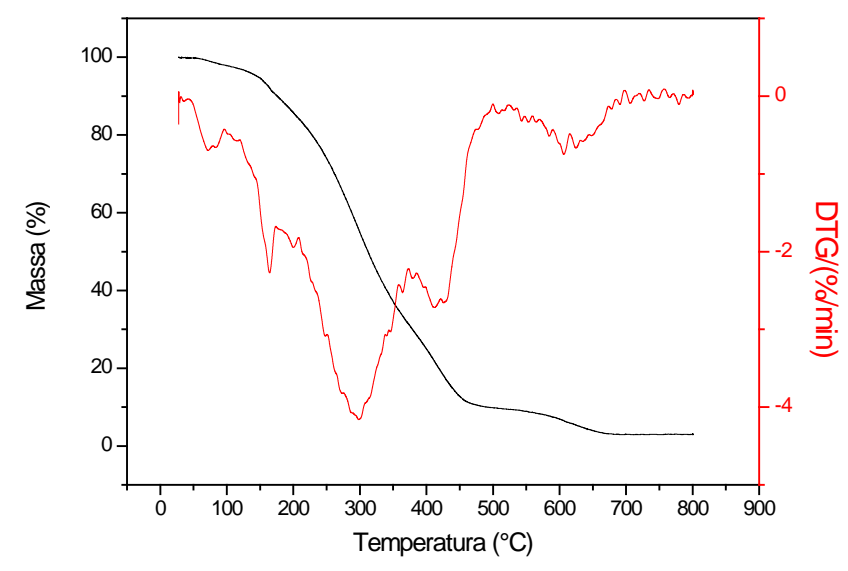

Gráfico 4.TG/DTG do biodiesel produzido com auxílio do microondas

A partir do gráfico é possível identificar cinco perdas de massa, onda a primeira se inicia a 46 ${ }^{\circ} \mathrm{C}$ e termina em $96^{\circ} \mathrm{C}$ tendo seu pico máximo em $70{ }^{\circ} \mathrm{C}$, tal evento pode ser devido a presença de água que restou no processo de evaporação do solvente. Para o segundo pico que tem início na temperatura de $96{ }^{\circ} \mathrm{C}$ e vai até $-174^{\circ} \mathrm{C}$ com seu valor máximo em $165^{\circ} \mathrm{C}$ é atribuída a formação de biodiesel logo o mesmo não estava presente na análise térmica realizada apenas para a matéria prima (Gráfico 1), o terceiro pico e de maior intensidade tendo início em uma temperatura de $174{ }^{\circ} \mathrm{C}$ e fim a $371{ }^{\circ} \mathrm{C}$ é atribuído ao material lipídico não reagido durante o processo de transesterificação (BARROS, 2015). Ao quarto pico na qual vai de de $371{ }^{\circ} \mathrm{C}$ a $514{ }^{\circ} \mathrm{C}$, Barros 2015, atribui a presença de compostos orgânicos de maiores massas molares não reagido. E o quinto e último pico atribuímos a compostos de maiores massas não identificado. Para melhor entender os eventos a Tabela 4 tem as principais informações da análise realizada.

Tabela 4- Faixa de temperatura onde se ocorre as principais perdas de massa. Para o biodiesel produzido

\begin{tabular}{cccccc}
\hline AMOSTRAS & EVENTOS & $\mathrm{T}\left({ }^{\circ} \mathrm{C}\right)$ & Tmáx. $\left({ }^{\circ} \mathrm{C}\right)$ & $\begin{array}{c}\text { PERDA DE } \\
\text { MASSA (\%) }\end{array}$ & $\begin{array}{c}\text { MASSA } \\
\text { RESIDUAL }\end{array}$ \\
\hline \multirow{3}{*}{ Biodiesel } & 1 & $46-96$ & 70 & 2 & \\
& 2 & $96-174$ & 165 & 17 & 93 \\
& 3 & $174-371$ & 298 & 45 & \\
& 4 & $371-514$ & 418 & 26 & \\
\hline
\end{tabular}

Quando comparando os dois métodos de extração de biodiesel, vale chamar atenção para as vantagens que o método com o uso do forno microondas apresenta. Primeiramente o tempo total de reação, que é bem menor do que o tempo necessário para que a transesterificação pelo método convencional ocorra, a mesma necessita de aproximadamente 4 horas de reação enquanto para a reação com o uso do forno microondas necessitou-se de apenas 30 minutos, a mesma por sinal ainda apresentou resultados de conversão bastante animador.

\section{CONCLUSÕES}

Conclui-se que a transesterificação in situ ácida se mostrou adequada já que seu propósito era de obtenção de biodiesel. Para a reação no microondas apresentou resultados de conversão do material 
em biodiesel bastante interessante e satisfatório, a reação realizada no mesmo se apresenta como um meio interessante para a síntese de biodiesel visto que o tempo de reação é menor do que o tempo necessário para a reação convencional. O método com o auxílio do microondas teve resultados bastante semelhantes utilizando tempo de reação consideravelmente menor, quando a reação com auxilio deste método utilizou apenas 30 minutos a convencional usou 4 horas os valores de conversão foram de 1 e 18,66 \%.

Use of sewage scum as raw material for biodiesel production using the microwave technique

\begin{abstract}
The need to develop new sources of cleaner energy and lower cost, has created an incentive for the production of biodiesel to be a source of renewable energy. The sewage scum is presented as a good option for such action as it may be found in the sewage treatment plants in large quantities. This work aims to obtain biodiesel through sewage scum using the transesterification method in situ traditional, with the help of the microwave. At first part of scum was submitted to the Bligh \& Dyer method for extracting the lipid material (ML). The conventional transesterification process was carried out for 4 hours at a temperature of $70^{\circ} \mathrm{C}$ using 10 grams of the dry foam $50 \mathrm{~mL}$ hexane, $150 \mathrm{~mL}$ ethanol and $5 \% \mathrm{H}_{2} \mathrm{SO}_{4}$, the same quantities of reagents had been used for the reaction in a microwave, with one exception where the total time of the reaction was reduced from 4 hours to 30 minutes with constant stirring, and then the material was taken to a separatory funnel and separated solvent extracted material finally biodiesel obtained was taken to oven for eventual separation solvent present in the environment. After extraction were made the characterization analysis, thermal analysis by TG and DTG. The model of the equipment used is the thermal analyzer STA brand 449F3-J upiter. The method of transesterification in situ with acid catalysis has been successfully performed, the same extraction technique proves to be effective in converting the biodiesel scum, as well as the reaction performed with the aid of microwave proved very promising.
\end{abstract}

Keywords: Transesterification situ; biodiesel; scum; Microwave.

\title{
REFERÊNCIAS BIBLIOGRÁFICAS
}

BARROS, H.S.; MAIA, C.E.M.; SOUZA, L.Z. Extração do óleo presente na escuma de esgoto para uso na produção de biodiesel. Revista Química: Ciência, Tecnologia e Sociedade. v. 2, p. 42-48, 2013.

BARROS, H.S. Uso de resíduos sólidos de uma estação de tratamento do esgoto-ete, para a produção de biodisel via catalise acida in situ. 2015. P.110 Dissertação- Universidade do Estado do Rio Grande do Norte.

CHRISTOFF, P. Produção de biodiesel a partir do óleo residual de fritura comercial. Estudo de caso: Guaratuba, litoral paranaense. Dissertação (Desenvolvimento de Tecnologia). Institutor de Tecnologia para o Desenvolvimento - LACTEC. Curitiba. 2006.

DWECK,J . SAMPAIO, C.M.S. Analysis of the thermal decomposition of commercial vegetable oils in air by simultaneous TG/DTA. J ournal of Thermal Analysis and Calorimetry. v.75, p. 985-391, 2004.

SHETALWY, S. T. E.; SHAKINAZ, A. E.; SHERBI NY, REFAAT, A. A. Production of biodiesel using the microwave technique. Journal of Advanced Research. V1. p. 309-314, 2010.

FIGUEIROA, D. S. et al. Diferentes rotas de síntese destinadas a obtenção de peneiras moleculares do tipo Al-SBA-15. 56 ${ }^{\circ}$ congresso Brasileiro de Cerâmica. p. 686-695 2012.

GARCIA, C.C. et al. Thermal stability studies of some cerrado plant oils. J ournal Termal Analysis Calorimetry. v. 87, p. 645-648, 2007. 
GOMES, A.F. Extração e caracterização do material lipídico e biodiesel microalgal proveniente da Monoraphidium sp. Dissertação (Mestrado de Química). Universidade Federal do Rio Grande do Norte - UFRN. Natal. 2013.

KARGBO, D.M. Biodiesel productionfrom municipal sewagesludges. Energy \& Fuels. v. 24. p. 27912794, 2010.

MONDALA, A. et al. Biodiesel prodction by in situ transesterication of municipal primary and secondary sludges. Revista Bioresource Technology, v. 100, p. 1203-1210, 2009.

OLIVEIRA, M. S. M.; BIESEK, L.; PERGHER, S. B. C.; Estudo dasintese e caracterização de uma Al/SBA-15 através do método de ajuste de pH. XEncontro Brasileiro sobre Adsorção. 2014 p. 1-7.

OLIVEIRA, L. B. Potencial de aproveitamento energético de lixo e de biodiesel de insumos residuais no Brasil. Tese (Doutorado em Ciências). Universidade Federal do Rio de Janeiro - UFRJ. 2004.

POKOO-AI KI NS, G. et al. A multi-criteria approach to screening alternatives for converting sewage sludge to biodiesel. J ournal of Loss Prevention in the Process Industries, v.23, p. 412-420, 2010.

SANTOS, A.G.D. Avaliação da estabilidade térmica e oxidativa do biodiesel de algodão, girassol, dendê e sebo bovino. Dissertação (Mestrado em Química) Universidade Federal do Rio Grande do Norte-UFRN. Natal, 2010.

YUAN, H.; YANG, B. L.; ZHU, G. L. Synthesis of Biodiesel Using Microwave Absorption Catalysts. Energy \& Fuels, p. 548- 552, 2009.

HUANG, K.; ZHU, H.; WU, L. Thermal analysis on the process of microwave-assisted biodiesel production. Bioresource Technology, p. 279 - 284, 2013. 